\title{
Erratum to: BMF: An Indexing Structure to Support Multi-element Check
}

\author{
Chenyang $X u^{(\bowtie)}$, Qin Liu, and Weixiong Rao \\ Department of Software Engineering, Tongji University, Shanghai, China \\ \{1336321, qin.liu,wxrao\}@tongji.edu.cn
}

\section{Erratum to: \\ Chapter 34: B. Cui et al. (Eds.) \\ Web-Age Information Management \\ DOI:10.1007/978-3-319-39937-9_34}

In an older version of the paper starting on page 441 of this volume, the name and email address of the second author (Qin Liu) were missing. This has been corrected. 